\title{
A Change of Heart: Human Cardiac Tissue Engineering as a Platform for Drug Development
}

\author{
Samantha B. Bremner ${ }^{1,5} \cdot$ Karen S. Gaffney $^{1} \cdot$ Nathan J. Sniadecki $^{1,2,3,4,5} \cdot$ David L. Mack $^{1,5,6} \oplus$
}

Accepted: 20 January 2022 / Published online: 5 March 2022

(c) The Author(s) 2022

\begin{abstract}
Purpose of Review Human cardiac tissue engineering holds great promise for early detection of drug-related cardiac toxicity and arrhythmogenicity during drug discovery and development. We describe shortcomings of the current drug development pathway, recent advances in the development of cardiac tissue constructs as drug testing platforms, and the challenges remaining in their widespread adoption.

Recent Findings Human pluripotent stem cell-derived cardiomyocytes (hPSC-CMs) have been used to develop a variety of constructs including cardiac spheroids, microtissues, strips, rings, and chambers. Several ambitious studies have used these constructs to test a significant number of drugs, and while most have shown proper negative inotropic and arrhythmogenic responses, few have been able to demonstrate positive inotropy, indicative of relative hPSC-CM immaturity.

Summary Several engineered human cardiac tissue platforms have demonstrated native cardiac physiology and proper drug responses. Future studies addressing hPSC-CM immaturity and inclusion of patient-specific cell lines will further advance the utility of such models for in vitro drug development.
\end{abstract}

Keywords Cardiac tissue engineering $\cdot$ Pluripotent stem cells $\cdot$ Drug screening $\cdot$ Cardiotoxicity

This article is part of the Topical Collection on Regenerative Medicine

David L. Mack

dmack21@uw.edu

Samantha B. Bremner

sbremner@uw.edu

Karen S. Gaffney

ksgafney@uw.edu

Nathan J. Sniadecki

nsniadec@uw.edu

1 Department of Bioengineering, University of Washington, Seattle, WA, USA

2 Department of Mechanical Engineering, University of Washington, Seattle, WA, USA

3 Department of Laboratory Medicine and Pathology, University of Washington, Seattle, WA, USA

4 Center for Cardiovascular Biology, University of Washington, Seattle, WA, USA

5 Institute for Stem Cell and Regenerative Medicine, University of Washington, Seattle, WA, USA

6 Department of Rehabilitation Medicine, University of Washington, Seattle, WA, USA

\section{Introduction}

The current drug development pathway is exorbitantly expensive and time-consuming. It is estimated that between 2009 and 2018, the median cost to bring a new drug to market was $\$ 985.3$ million, including capitalized research and development investment costs [1]. This figure includes the cost of failed trials, as it has been reported that the approval rate for drugs entering phase I trials is only $13.8 \%$ [2]. Even having reached later phases of clinical trials, it is unfortunately common for drugs to fail due to lack of efficacy or unanticipated adverse effects, regardless of the promise shown in preclinical studies [3]. In a large part, this is due to limitations of animal models and in vitro preclinical models that limit our ability to accurately predict drug performance in human patients $[4,5]$. Additionally, many pharmaceutical companies opt to embrace a fail early, fail fast strategy where the vast majority of drug candidates are eliminated early in the process to avoid costly late-stage failures, thus possibly missing potentially life-saving drugs [6]. In light of this, it is necessary that we re-evaluate the preclinical drug discovery and testing paradigm to make drug development more efficient and cost effective. 
The shortcomings of the canonical drug development process are perhaps most evident when considering cardiac effects, as side effects such as cardiac toxicity and arrhythmogenicity are the most common reasons for latestage drug failure or drug withdrawal [7, 8]. To address this, regulatory agencies require specific cardiotoxicity screening; however, the methods employed lack the relevance to the human cardiovascular system needed to accurately predict cardiac effects. The more simple in vitro models rely on non-cardiac cell lines that express a recombinant human ether-á-go-go related gene (hERG) potassium channel, as this channel plays a major role in cardiac repolarization and hERG-blocking compounds frequently cause ventricular arrhythmias $[9,10]$. While useful in some cases, these simple, in vitro models are unable to model the interactions of multiple ion channels and the compensatory mechanisms present in the native myocardium. Increasing in complexity, rodent models are frequently used due to their relative low costs and short experimental timelines. However, these models often produce irrelevant results due to distinct physiological differences between human and rodent cardiovascular biology $[5,11]$. While larger animal models can yield more relevant results, the costs and resources required for colony maintenance and ethical concerns are often prohibitive [12]. Thus, there exists a need to develop improved human in vitro cardiac models for drug screening and development.

The human heart is the ideal platform for drug testing; however, the limited availability and inadequate ex vivo viability of primary samples prevents their widespread use. Consequently, the development of human pluripotent stem cells (hPSCs, either embryonic stem cells, hESCs, or induced pluripotent stem cells, hiPSCs) and hPSC-derived cardiomyocytes (hPSC-CMs) holds great promise for advancing drug development platforms [13-16]. To date, hPSC-CMs have been shown to express key cardiomyocyte structural and signaling elements and faithfully recapitulate human cardiac biology, leading to their widespread use in studies demonstrating typical responses to drug compounds. However, hPSC-CMs are limited in their relative immaturity as compared to adult cardiomyocytes, as hPSC-CMs have been shown to more closely resemble fetal cardiomyocytes with regard to transcriptional activity, ultrastructure, and function, limiting their utility as a preclinical drug screening model [17-19]. Thus, several approaches have been employed to improve hPSC-CM maturity, including increased time in culture, topographical cues, biochemical stimuli, and the development of three-dimensional engineered models [20].

Human engineered cardiac tissues can be broadly defined as multicellular aggregates made from hPSCCMs, often accompanied by other cell types, with or without the presence of extracellular matrix protein scaffolds. Such engineered constructs more closely mimic the native myocardium by recapitulating key cell-cell and cell-matrix biology that has been shown to further advance hPSC-CM maturation and facilitate key measures of cardiac function such as force production and voltage propagation [21-25]. These tissue constructs come in many shapes and sizes ranging from scaffold-free spheroids amenable to high-throughput screening to larger engineered chambers capable of generating pressure-volume loops. In recognition of the promise of these cardiac constructs to improve our ability to model cardiomyopathy and drug responses in vitro, research groups have developed various platforms, and many have moved toward commercialization. This review describes the newest advances made in the development of engineered cardiac constructs as valid platforms for preclinical drug screening and the remaining challenges preventing widespread adoption of these platforms.

\section{Engineered Cardiac Platforms for Drug Screening}

As a category, engineered cardiac tissues have come to include any cell culture platform facilitating multicellular, three-dimensional culture of synchronously contracting hPSC-CMs. As such, these platforms take many forms that vary widely in geometry and scale, the inclusion of nonmyocytes, and the presence of scaffold proteins. Broadly, those on the smaller scale such as spheroids and microtissues more easily facilitate high-throughput cardiotoxicity screening while retaining some aspects of cardiac function, whereas larger platforms including cardiac sheets, strips, rings, and chambers are more suited to lower-throughput assessment of drug effect on cardiac function, as they more closely resemble native cardiac tissue and enable measurement of voltage propagation and force generation (Fig. 1). In the following sections, we have categorized these platforms broadly by geometry as a means to discuss their advantages and limitations as platforms for drug testing and progress made toward drug screening applications. Details describing the various platforms and findings from recent drug screening studies are summarized in Table 1.

\section{a. Spheroids}

At the smallest end of the scale are cardiac spheroids, also often referred to as cardiac organoids. Cardiac spheroids are small hPSC-CM aggregates that are formed by hanging-drop [26] or self-assembly on lowattachment substrates [27]. These platforms are generally scaffold-free, which allows for a dense network of cell-cell connections and removes any concern of drug absorption by scaffolding hydrogels or silicone support structures often used for larger platforms [28]. Spheroid 


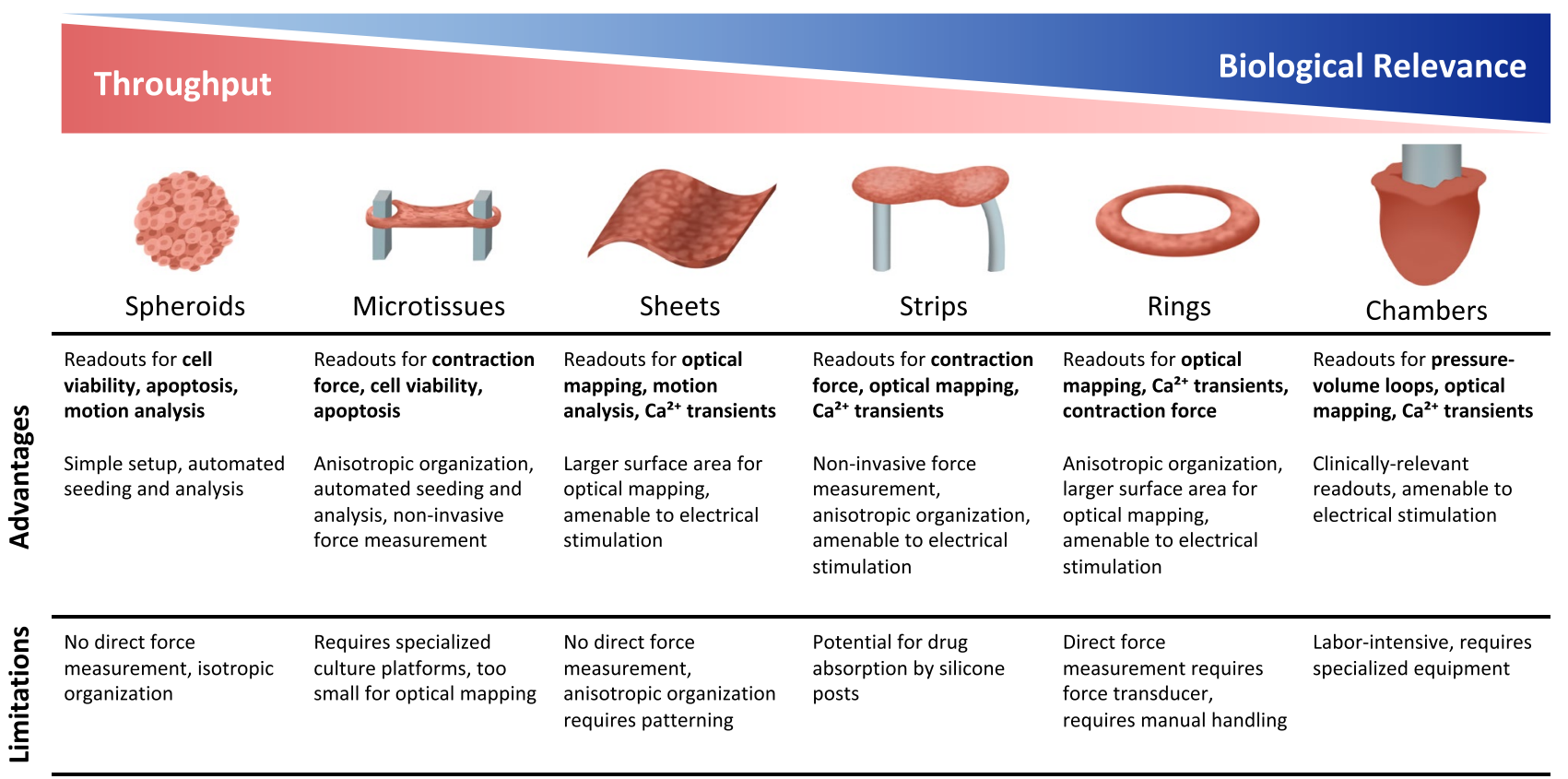

Fig. 1 Different engineered cardiac tissue platforms organized by relative throughput and biological relevance with a description of their advantages and limitations

systems often include non-myocyte cell types such as endothelial, fibroblast, and mesenchymal cells, which has repeatedly been shown to enhance spheroid function [29-31]. Perhaps most attractively, the small scale of cardiac spheroids requires significantly lower resources in terms of cell number and culture space and is most easily amenable to automated generation and high-throughput analysis.

Cardiac spheroids have been used extensively to develop drug testing platforms. In an earlier study, it was shown that cardiac spheroids generated from hiPSCCMs were similarly able to model doxorubicin-mediated cardiotoxicity as compared to spheroids made from primary human cardiomyocytes [32]. Demonstrating their high-throughput capabilities, a study used cardiac spheroids to screen a panel of 29 compounds approved by regulatory agencies with or without known structural cardiotoxicity [33•]. It was demonstrated that this platform was able to detect changes in cellular viability, endoplasmic reticulum integrity, and mitochondrial membrane potential. Beyond structural effects, cardiac spheroids have been used to stratify pro-arrhythmic toxicity of hERG channel blockers and environmental toxins [34].

Using cardiac spheroids, it is also possible to model disease states. To model cardiac fibrosis, spheroids generated from hESC-CMs and hESC-derived mesenchymal stem cells were treated with transforming growth factor beta (TGF- $\beta$ ) [31]. It was shown that TGF- $\beta$ triggered fibrotic features in the cardiac spheroids and that this response was worsened with the administration of known cardiotoxins. In a separate study, cardiac spheroids were used to model myocardial infarction by culturing in hypoxic conditions and treating with noradrenaline [35]. It was shown that hypoxic conditions worsened doxorubicin-mediated cardiotoxicity, while an antifibrotic compound could reduce ischemic spheroid stiffness and asynchronicity.

While the use of cardiac spheroids for drug screening is advantageous given their relative accessibility and high-throughput nature, these culture systems do not promote uniaxially aligned contractile machinery, and functional outputs are often limited to cell viability with some insight into contractility and arrhythmogenicity by measurement of spheroid deflection. However, it is possible for spheroids to serve as building blocks for higher order tissues, as was demonstrated with bioprinting of spheroids into larger cardiac rings [36]. Ultimately, higher-throughput spheroid systems may be best suited for earlier stages of drug compound testing.

b. Microtissues

To increase functional readout capacity while retaining the high-throughput benefits of spheroid culture systems, many have developed what we here call microtissues, where a similarly small number of cells, sometimes in a hydrogel scaffold, are self-assembled onto manufac- 


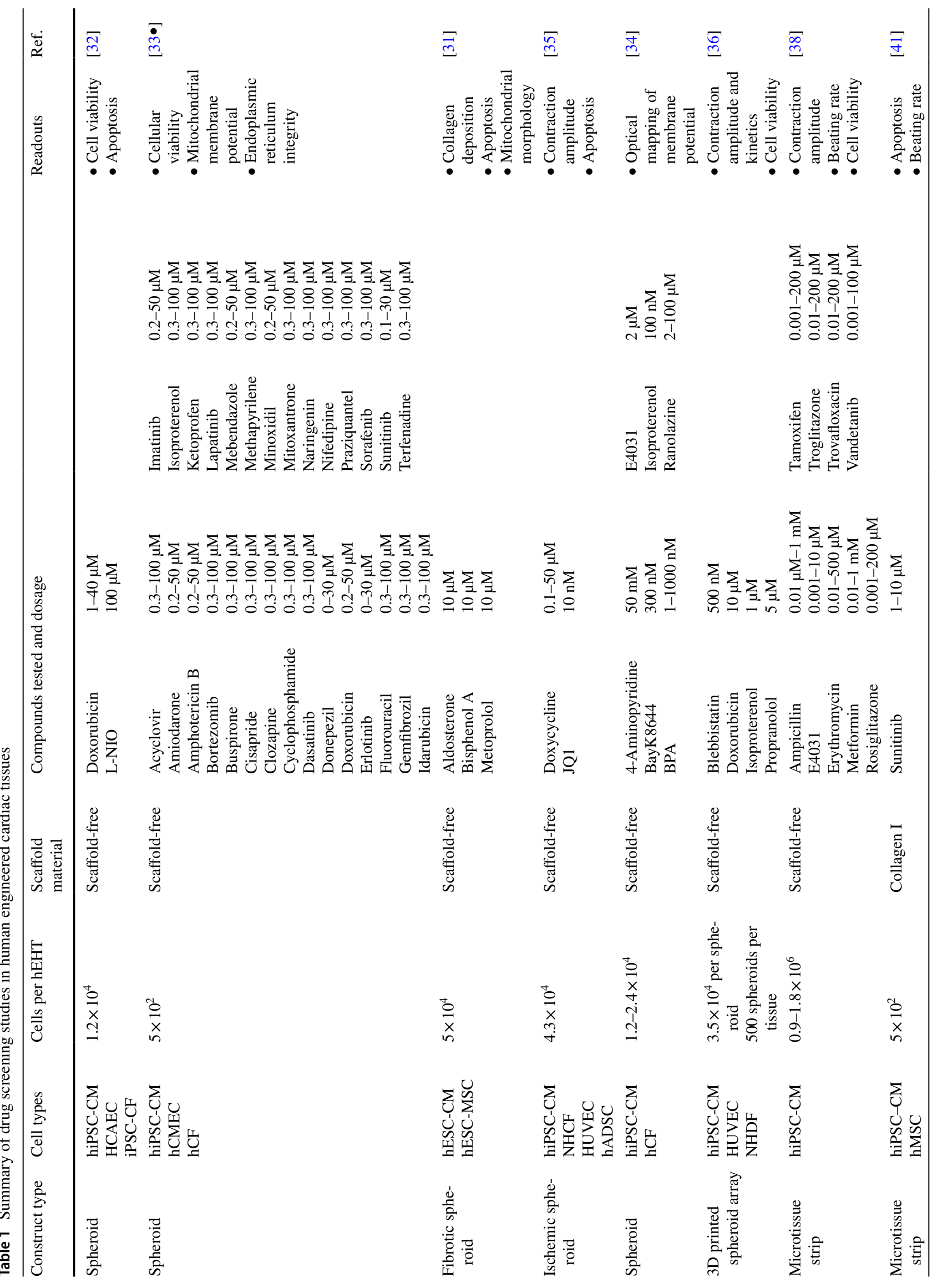




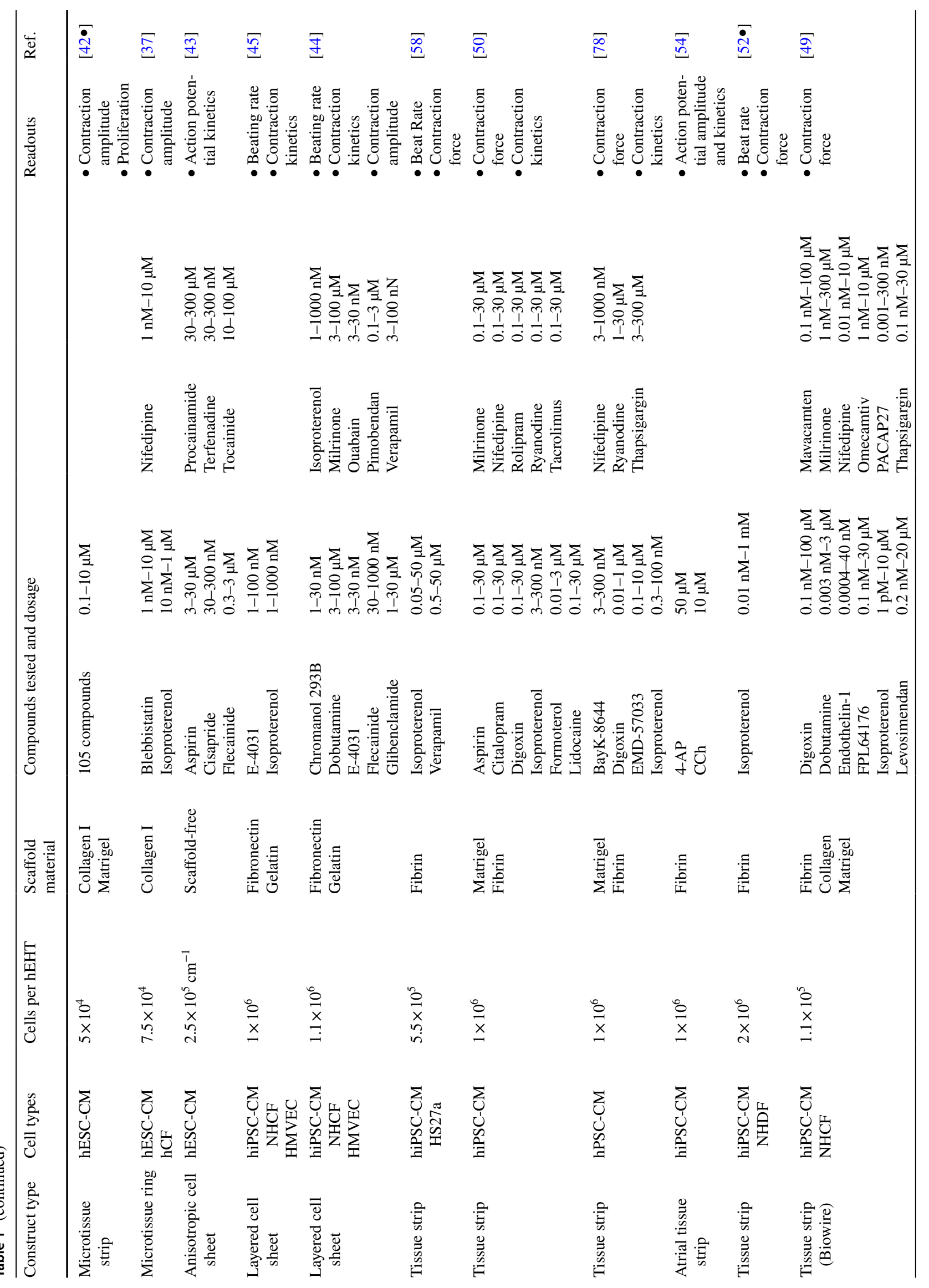




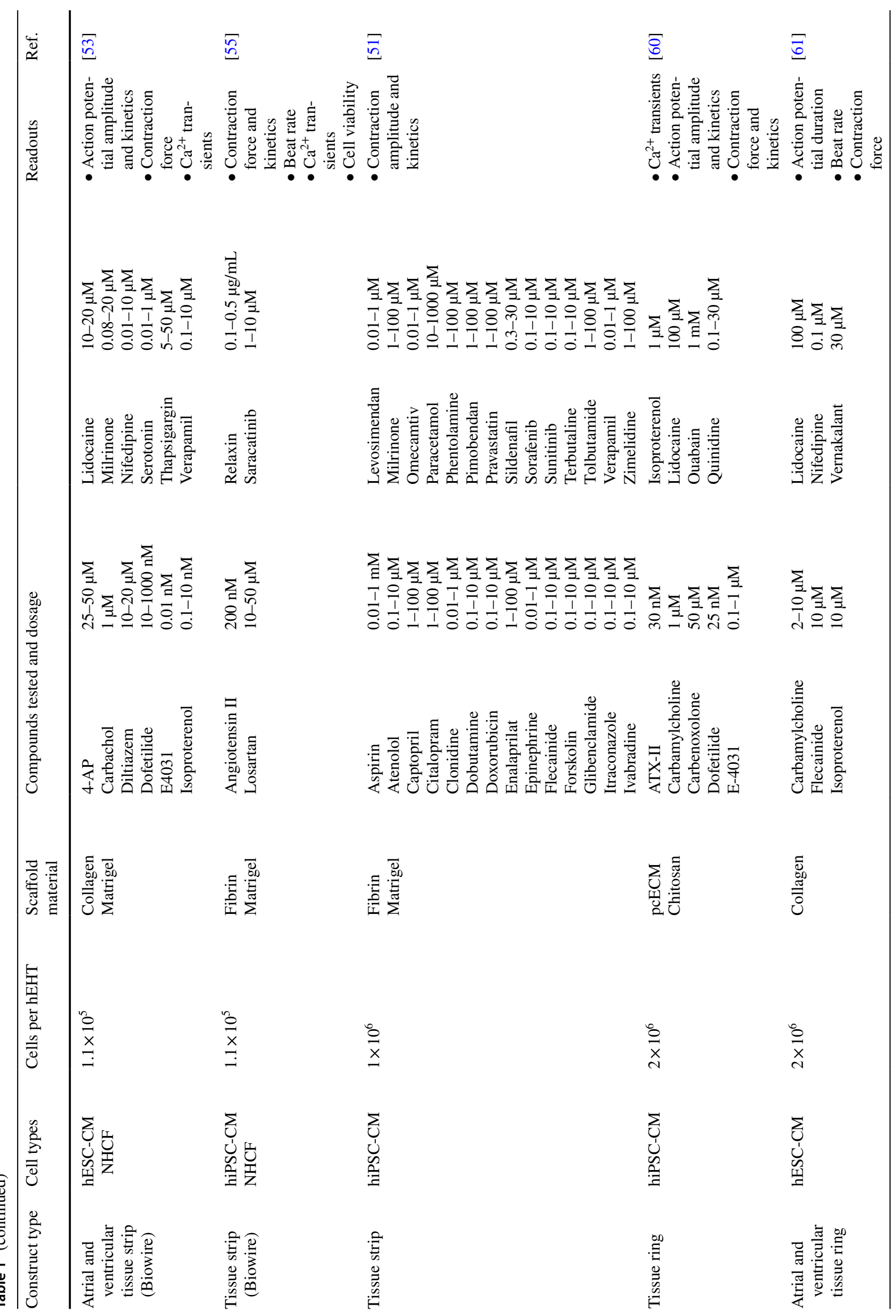




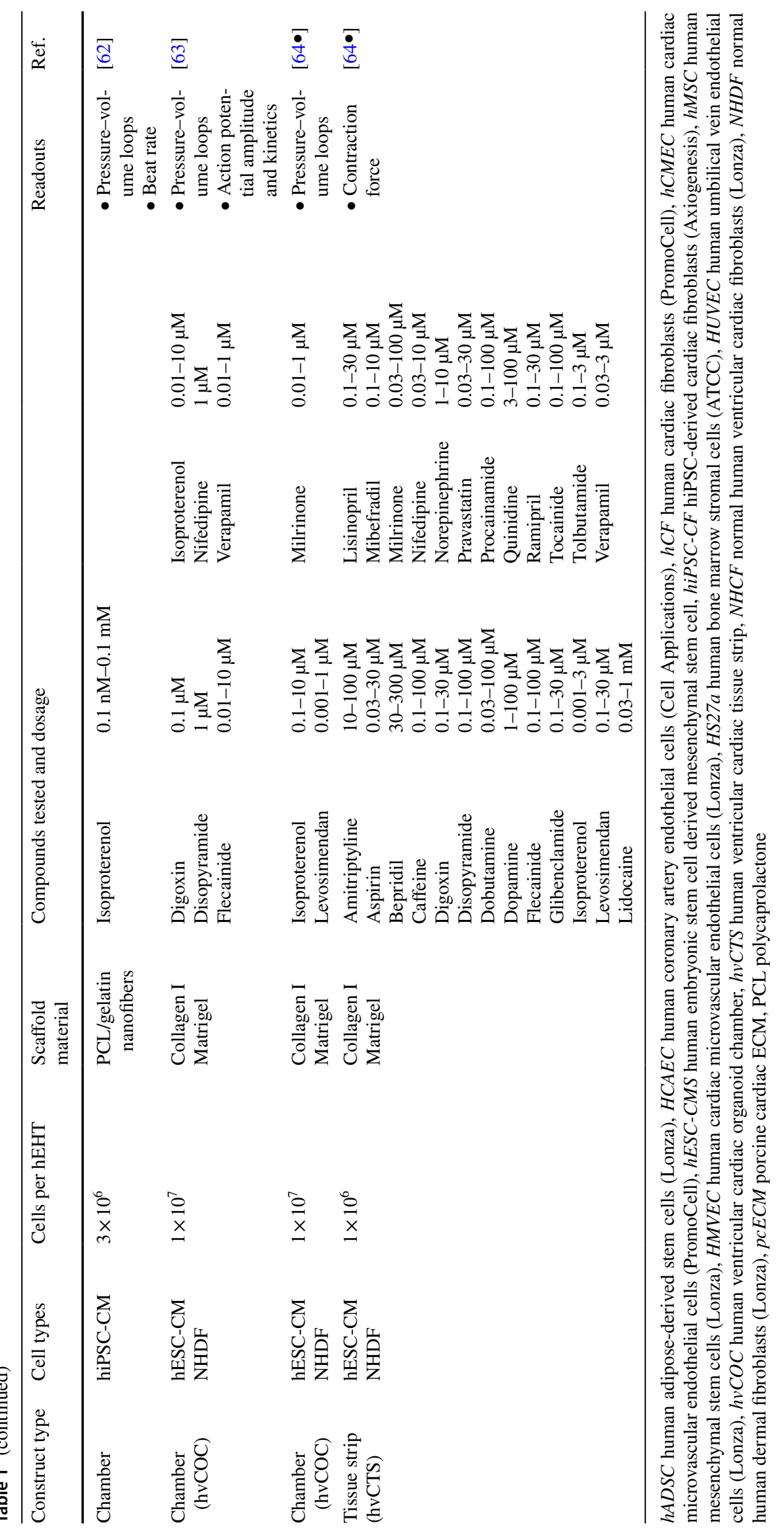


tured posts, such that they form geometries similar to the cardiac strips and rings presented in the following sections [37-41]. It was demonstrated that microtissues outperform age-matched two-dimensional hiPSC-CMs in terms of predictive accuracy in drug response [38]. Building on the high-throughput advantages of spheroid culture systems, these microtissue platforms provide uniaxial mechanical cues, generating improved cellular alignment and facilitate more rigorous measurement of contractile function by tracking the deflection of cantilever posts.

Possibly the most useful application of microtissues or other smaller cardiac platforms is as an intermediate screen between high-throughput two-dimensional in vitro experiments and animal studies. This paradigm was demonstrated in a study screening for pro-proliferative compounds using 96-well microtissues, where an initial pool of approximately 5,000 compounds were screened in twodimensional hiPSC-CMs for their ability to induce cellular proliferation [42•]. Of this initial pool, 105 compounds were identified and screened further for pro-proliferative effects using microtissues, which also allowed for the elimination of compounds causing negative functional effects. A smaller pool of the leading compounds were then further evaluated in microtissues that were further matured with fatty acid supplementation [40, 42•]. This study uniquely demonstrates a pathway by which to pursue drug development with varying hierarchies of in vitro hPSC-CM models.

\section{c. Sheets}

Cardiac sheets consist of one or multiple layers of hPSC-CMs and are particularly useful for detecting arrhythmogenicity. Using fluorescent voltage or calciumsensitive dyes or genetically encoded sensors, it is possible to model arrhythmic risk of drug compounds by visualizing conduction speeds and re-entry waves as was done in two-dimensional cell sheets [43]. This platform has the additional benefit of microgrooves providing anisotropic cell patterning that more closely represents native myocardium. Other groups have developed methods of coating hPSC-CMs and other cell types with ECM and seeding them into cell sheets that are multiple layers thick $[44,45]$. Using motion tracking, it was possible to measure the effects of several drug compounds on magnitude of contraction, contraction kinetics, and abnormal beat intervals [44]. However, shortcomings of cardiac sheets include the difficulty in obtaining direct measurement of force output and the need for additional interventions to facilitate sheet patterning and cellular alignment.

\section{d. Tissue Strips}

Cardiac strips are perhaps the most commonly thought of hEHT platform along with spheroids and are made from hPSC-CMs embedded in a hydrogel that is cast into a mold where it solidifies and subsequently compacts and begins beating spontaneously [46]. Cardiac strips are cast uniaxially between two elastomeric posts [47] or wires (Biowire) [48, 49]. This platform enables higher-throughput measurement of contractile forces via tracking the deflection of elastomeric supports. As such, these models are particularly suited to drug screening, where easily measured force production and kinetics can provide insights into the inotropic and arrhythmogenic effects of test compounds.

To date, cardiac strips have been used to test a wide panel of compounds with and without known cardiac effects. A panel of eleven compounds was tested on cardiac strips, demonstrating that these tissues were able to faithfully reproduce positive and negative inotropic effects when compared to human atrial trabeculae, but the relative immaturity of the hiPSC-CMs was evident due to limited observed beta-adrenergic effects [50]. The benefits of three-dimensional culture were further demonstrated in another study where an impressive panel of 28 drugs was tested on cardiac strips as well as 2D hiPSC-CM monolayers, where it was demonstrated that the tissues yielded more accurate drug responses in terms of contractility and calcium transient response (85\% accuracy for hiPSC-CM monolayers vs. 93\% accuracy for tissue strips) [51]. Additionally, both of these studies demonstrate the potential for increased throughput with tissue strip platforms despite their larger size, as not only were multiple drugs tested, but at multiple doses, enabling the derivation of EC50 values.

Chronic electrical stimulation of cardiac strips has been demonstrated to improve tissue maturity and promote positive force-frequency relationships, thus improving the accuracy of drug responses and the ability to model positive inotropy [52•]. Similar stimulation protocols have been used on the Biowire II platform, which was used to derive EC50 values and demonstrate canonical responses for several drug compounds [49]. This platform and others have been further developed to model specific atrial and ventricular responses to drugs in chamber-specific tissues $[53,54]$ and to explore antifibrotic drugs in angiotensin II-mediated non-genetic cardiomyopathy [55]. Despite their increased size and required resources as compared to spheroids or microtissues, strip hEHTs have been used to generate impressive datasets demonstrating relevant responses to many com- 
pounds at various doses in a single study. Paired with efforts to improve hEHT maturity, efforts to increase analysis throughput through optical $[56,57]$ or magnetic detection of post movement [58], will only further increase the utility of cardiac strip platforms.

e. Tissue Rings

Cardiac rings are similar to cardiac strips save for their shape, where cardiac rings are cast in circular molds before being transferred to isometric or elastomeric supports [59]. Given their larger surface area, cardiac rings are particularly suited to modeling voltage propagation as an indicator of arrhythmogenicity. Using genetically encoded voltage and calcium sensors, tissue rings have been shown to properly model drug-induced changes in contraction rate and conduction properties [60]. Additionally, by using patient-derived hiPSCs, authors were able to reproduce long QT syndrome and demonstrate drug-induced reentrant arrhythmias. This platform was further developed to model chamber-specific responses to a panel of drugs by using atrial or ventricular hiPSCCMs [61]. While tissue ring platforms are useful for modeling drug-induced changes in conduction properties and arrhythmogenicity, it would appear that they may be less popular than other platforms of similar size. This may be due to the requirement for more individual tissue handling, as measurement of contractile forces often requires the use of a force transducer, which hampers throughput.

\section{f. Chambers}

At the opposite end of the spectrum from cardiac spheroids are cardiac chambers, which resemble a miniaturized ventricle $[62,63]$. The geometry of this platform most closely resembles a native ventricle, and engineered cardiac chambers are the only platform capable of generating pressure, enabling measurement of clinically relevant outputs including ejection fraction, cardiac output, and pressure-volume loops. Cardiac chambers have been shown to surpass lower-order 2D and 3D hPSC-CM culture systems in transcriptional maturity [63]. Given their advanced maturation and attainable performance metrics, chamber constructs are an appealing platform for drug screening and characterization.

One such platform (human ventricle-like cardiac organoid chamber, hvCOC) is generated by casting hESC$\mathrm{CMs}$ in a hydrogel around a balloon catheter, which is removed after tissue compaction [63]. This initial study demonstrated altered pressure-volume loops and electrophysiological performance after treatment with six compounds. In a follow-up study, hvCOCs and human ventricular-like cardiac tissue strips (hvCTS) were treated with a panel of 25 cardioactive compounds, where it was demonstrated that hvCOCs displayed enhanced positive inotropy as compared to hvCTSs [64•]. A similar platform has been developed using pullspun nanofibers that recapitulate the concentric, anisotropic orientation of native myocardium [62]. While it was possible to measure pressure-volume loops, this model failed to replicate a positive inotropic response with isoproterenol treatment, indicating relative immaturity.

Compared to other cardiac tissue platforms, cardiac chambers are limited in terms of the increased resources and technical expertise required, ultimately resulting in a lower-throughput platform. However, with the addition of additional maturation techniques such as electrical stimulation and anisotropic cell sheet patterning [65], such models could effectively serve as in vitro replacements for Langendorff whole-heart preparations.

\section{Challenges Remaining}

Despite wide-spread excitement surrounding engineered cardiac platforms for drug development and several commercialization efforts underway, several hurdles remain. Compared to adult myocardium, or even neonatal myocardium, engineered cardiac constructs present a very immature phenotype, potentially limiting their physiological relevance. In early stages after differentiation with no intervention, hPSC-CMs display only fetal transcriptomes [66], ion channel expression [67], metabolic function [68], and contractility [69]. While three-dimensional culture has been shown to promote advanced hPSC-CM maturation [21-25], it is evidently insufficient to routinely produce a robust cardiac phenotype that includes positive force-frequency responses and positive inotropic responses, thus potentially limiting their use as drug screening platforms. Several methods shown to advance hPSC-CM maturity in two-dimensional culture could potentially have the same effect in tissues, including fatty acid [70] or thyroid hormone supplementation [71] or microRNA treatment [72, 73]. Advanced engineering approaches have been employed to further increase the maturity achieved in cardiac tissues, including electrical stimulation [52•], increasing afterload [74], and the addition of preload or passive stretch $[75,76]$, which, if successful, will greatly improve the predictive capacity of these engineered models.

A significant difficulty that has become apparent when working with engineered tissues is controlling variability and demonstrating reproducibility. This variability arises, in part, from hPSC-CM batch-to-batch variability and the different protocols used by different institutions for hPSC-CM 
differentiation and tissue generation, both of which are inherently human processes where results may vary simply by the hands performing the experiment. To remove human sources of variability, many are turning toward automation of tissue generation and analysis, which will also increase platform throughput $[37,57,77]$. Additional variation arises from the different genetic backgrounds of the various hPSC lines used. Highlighting this challenge, a study compared the performance of ten different hPSC lines in cardiac tissue strips [78]. It was found that spontaneous and electrically paced tissue contractile performance and kinetics varied widely between the different lines, emphasizing the need for isogenic controls in disease modeling and advocating for the use of multiple hPSC lines during platform validation. Interestingly, it was found that despite the variability in baseline performance, the different hPSC lines behaved more consistently with regard to drug response, although with varying EC50 values [78]. To address this concern, it is likely that robust cardiac tissue platforms will continue to rely on multiple biological replicates and turn toward automation and the use of multiple genetic backgrounds.

Conversely, this variability can be seen as a facet of hPSC and tissue engineering that has not yet been fully taken advantage of. There are numerous studies describing the development of hPSC lines harboring cardiomyopathyassociated mutations that could be further used to screen disease-specific drug candidates in engineered cardiac constructs [79]. Additionally, such engineered tissue models can be used to examine biological sex-related differences in cardiac biology and disease, as these differences are known, but often overlooked in in vitro disease modeling and preclinical screening [80]. By including multiple cell lines from different genetic backgrounds and with different disease-causing genetic variants, we can begin to approximate not only personalized and patient-specific medicine, but also populationwide responses to different pharmacological agents in the dish.

A significant limitation to translatability is that most platforms lack the biological complexity needed to fully reproduce native myocardium, necessitating the continued reliance on animal models for preclinical testing. At their simplest, cardiac constructs contain only hPSC-CMs, while others have included additional cell types including fibroblasts, endothelial cells, and mesenchymal cells, which have been shown to improve tissue quality and maturity. A biological element often missing from engineered cardiac constructs is vasculature, a key component needed to accurately model drug delivery. Strategies to vascularize constructs consist of co-culturing with endothelial cells and addition of angiogenic factors, three-dimensional bioprinting, or microfluidic systems [81, 82]. The development of in vitro models that better recapitulate the complexity of drug delivery and toxicity will also require the inclusion of additional organ systems involved in drug metabolism and clearance, e.g., hepatic and renal systems. To achieve this, several groups are developing complex, modular organon-a-chip systems [83, 84]. If successful, such vascularized multi-organ systems would be the pinnacle of in vitro drug testing platforms.

Lastly, it is worth noting that the vast majority of studies described in this review evaluated only compounds with known effects in human patients. As such, further studies are needed to demonstrate the true predictive capabilities of engineered cardiac tissues for clinical trial success. However, some pioneering studies have used engineered constructs to explore novel antifibrotic agents [55], perform screening experiments to identify pro-proliferative compounds for heart regeneration [42•], evaluate a novel myotrope [85], and even evaluate the effect of COVID-19 treatments on cardiac function [86]. Thus, it is evident that the stage is now set to explore the potential effects of novel therapeutics in engineered cardiac platforms.

\section{Conclusion}

In order to increase the efficiency with which new drugs are discovered and brought to clinical trials, it is necessary to improve the human in vitro models used, to increase their biological relevance and enable the field to move away from a reliance on animal models as the gold standard. To address concerns of cardiac toxicity, great efforts have been put toward the development of engineered cardiac tissues from hPSC-CMs. These platforms range in shape and scale from cardiac spheroids and microtissues, to sheets, strips, and rings, to chambers emulating an entire ventricle, all of which present their own advantages and limitations. This review has highlighted recent advances made in the development of cardiac tissue engineering for drug screening platforms. While lacking functional maturity and structural complexity in some regards, these constructs hold merit as drug screening platforms with powerful predictive capabilities that, as they stand, can provide value to early stages of the drug screening pipeline. With continued advancements in tissue maturity, automation, and throughput, it is our prediction that cardiac tissue engineering will continue to gain favor in the pharmaceutical industry.

\section{Declarations}

Conflict of Interest Samantha B. Bremner reports grants from National Science Foundation Graduate Research Fellowship (Grant No. DGE1762114), during the conduct of the study. Karen S. Gaffney has nothing to disclose. Nathan J. Sniadecki reports grants from the National Science Foundation (CMMI-1824792), National Institutes of Health 
(HL149734, TR003519, DE029827), and Sana Biotechnology during the conduct of the study and others from Curi Bio and Stasys Medical outside the submitted work. In addition, Dr. Sniadecki has a patent US20190029549A1 "System for magnetic detection of myocardial forces" licensed to Curi Bio. David L. Mack reports grants from Institute for Stem Cell and Regenerative Medicine Innovation Pilot Award, Senator Paul D. Wellstone Muscular Dystrophy Cooperative Research Center P50AR065139, and Jesse's Journey Foundation, during the conduct of the study, and others from Curi Bio, Inc. and Kinea Bio, Inc., outside the submitted work.

Human and Animal Rights and Informed Consent This article does not contain any studies with human or animal subjects performed by any of the authors.

Open Access This article is licensed under a Creative Commons Attribution 4.0 International License, which permits use, sharing, adaptation, distribution and reproduction in any medium or format, as long as you give appropriate credit to the original author(s) and the source, provide a link to the Creative Commons licence, and indicate if changes were made. The images or other third party material in this article are included in the article's Creative Commons licence, unless indicated otherwise in a credit line to the material. If material is not included in the article's Creative Commons licence and your intended use is not permitted by statutory regulation or exceeds the permitted use, you will need to obtain permission directly from the copyright holder. To view a copy of this licence, visit http://creativecommons.org/licenses/by/4.0/.

\section{References}

Papers of particular interest, published recently, have been highlighted as:

\section{- Of importance}

1. Wouters OJ, McKee M, Luyten J. Estimated research and development investment needed to bring a new medicine to market, 2009-2018. J Am Med Assoc. 2020;323(9):844-53. https://doi. org/10.1001/jama.2020.1166.

2. Wong $\mathrm{CH}$, Siah KW, Lo AW. Estimation of clinical trial success rates and related parameters. Biostatistics. 2019;20(2):273-86. https://doi.org/10.1093/biostatistics/kxx069.

3. Packer M. Unbelievable folly of clinical trials in heart failure. Circ Hear Fail. 2016;9(4). https://doi.org/10.1161/CIRCHEARTFAILURE. 116.002837.

4. Hartung T. Food for thought; look back in anger - what clinical studies tell us about preclinical work. Altex. 2013;30(3):275-91. https://doi.org/10.14573/altex.2013.3.275.

5. Olson H, Betton G, Robinson D, Thomas K, Monro A, Kolaja G, et al. Concordance of the toxicity of pharmaceuticals in humans and in animals. Regul Toxicol Pharmacol. 2000;32(1):56-67. https://doi.org/10.1006/rtph.2000.1399.

6. Paul SM, Mytelka DS, Dunwiddie CT, Persinger CC, Munos $\mathrm{BH}$, Lindborg SR, et al. How to improve R\&D productivity: The pharmaceutical industry's grand challenge. Nat Rev Drug Discov. 2010;9(3):203-14. https://doi.org/10.1038/nrd3078.

7. Onakpoya IJ, Heneghan CJ, Aronson JK. Post-marketing withdrawal of 462 medicinal products because of adverse drug reactions: a systematic review of the world literature. BMC Med. 2016;14(1):1-11. https://doi.org/10.1186/s12916-016-0553-2.

8. Laverty H, Benson C, Cartwright E, Cross M, Garland C, Hammond $\mathrm{T}$, et al. How can we improve our understanding of cardiovascular safety liabilities to develop safer medicines? Br J Pharmacol. 2011;163(4):675-93. https://doi.org/10.1111/J.1476-5381.2011. 01255.X.

9. Fernandez D, Ghanta A, Kauffman GW, Sanguinetti MC. Physicochemical features of the hERG channel drug binding site. J Biol Chem. 2004;279(11):10120-7. https://doi.org/10.1074/jbc.M310683200.

10. Gintant G, Sager PT, Stockbridge N. Evolution of strategies to improve preclinical cardiac safety testing. Nat Rev Drug Discov. 2016;15(7):457-71. https://doi.org/10.1038/nrd.2015.34.

11. Milani-Nejad N, Janssen PML. Small and large animal models in cardiac contraction research: advantages and disadvantages. Pharmacol Ther. 2014;141(3):235-49. https://doi.org/10.1016/J. PHARMTHERA.2013.10.007.

12. Silva KAS, Emter CA. Large animal models of heart failure: a translational bridge to clinical success. JACC Basic to Transl Sci. 2020;5(8):840-56. https://doi.org/10.1016/j.jacbts.2020.04.011.

13. Thomson JA. Embryonic stem cell lines derived from human blastocysts. Science (80- ). 1998;282(5391):1145-7. https://doi. org/10.1126/science.282.5391.1145.

14. Takahashi K, Yamanaka S. Induction of pluripotent stem cells from mouse embryonic and adult fibroblast cultures by defined factors. Cell. 2006;126(4):663-76. https://doi.org/10.1016/j.cell.2006.07.024.

15. Mummery CL, Zhang J, Ng ES, Elliott DA, Elefanty AG, Kamp TJ. Differentiation of human embryonic stem cells and induced pluripotent stem cells to cardiomyocytes: a methods overview. Circ Res. 2012;111(3):344-58. https://doi.org/10.1161/CIRCRESAHA. 110.227512 .

16. Lian X, Hsiao C, Wilson G, Zhu K, Hazeltine LB, Azarin SM, et al. Robust cardiomyocyte differentiation from human pluripotent stem cells via temporal modulation of canonical Wnt signaling. Proc Natl Acad Sci USA. 2012;109(27). https://doi.org/10.1073/pnas. 1200250109.

17. Snir M, Kehat I, Gepstein A, Coleman R, Itskovitz-Eldor J, Livne E, et al. Assessment of the ultrastructural and proliferative properties of human embryonic stem cell-derived cardiomyocytes. Am J Physiol - Hear Circ Physiol. 2003;285(6 54-6). https://doi.org/10.1152/ajpheart.00020.2003.

18. Synnergren J, Améen C, Jansson A, Sartipy P. Global transcriptional profiling reveals similarities and differences between human stem cell-derived cardiomyocyte clusters and heart tissue. Physiol Genomics. 2012;44(4):245-58. https:// doi.org/10.1152/physiolgenomics.00118.2011.

19. Tan SH, Ye L. Maturation of pluripotent stemcell-derived cardiomyocytes: a critical step for drug development and cell therapy. J Cardiovasc Transl Res. 2018;11(5):375-92. https:// doi.org/10.1007/s12265-018-9801-5.

20. Marchianò S, Bertero A, Murry CE. Learn from your elders: developmental biology lessons to guide maturation of stem cellderived cardiomyocytes. Pediatr Cardiol. 2019;40(7):1367-87. https://doi.org/10.1007/s00246-019-02165-5.

21. Mihic A, Li J, Miyagi Y, Gagliardi M, Li SH, Zu J, et al. The effect of cyclic stretch on maturation and 3D tissue formation of human embryonic stem cell-derived cardiomyocytes. Biomaterials. 2014;35(9):2798-808. https://doi.org/10.1016/j.biomaterials. 2013.12.052.

22. Ruan JLL, Tulloch NL, Razumova M V., Saiget M, Muskheli $\mathrm{V}$, Pabon L, et al. Mechanical stress conditioning and electrical stimulation promote contractility and force maturation of induced pluripotent stem cell-derived human cardiac tissue. Circulation. 2016;134(20):1557-67. https://doi.org/10.1161/ CIRCULATIONAHA.114.014998.

23. Ruan JL, Tulloch NL, Saiget M, Paige SL, Razumova MV, Regnier $\mathrm{M}$, et al. Mechanical stress promotes maturation of human myocardium from pluripotent stem cell-derived progenitors. Stem Cells. 2015;33(7):2148-57. https://doi.org/10.1002/stem.2036. 
24. Abilez OJ, Tzatzalos E, Yang H, Zhao MT, Jung G, Zöllner AM, et al. Passive stretch induces structural and functional maturation of engineered heart muscle as predicted by computational modeling. Stem Cells. 2018;36(2):265-77. https://doi.org/10.1002/stem.2732.

25. Shimko VF, Claycomb WC. Effect of mechanical loading on three-dimensional cultures of embryonic stem cell-derived cardiomyocytes. Tissue Eng - Part A. 2008;14(1):49-58. https:// doi.org/10.1089/ten.a.2007.0092.

26. Beauchamp P, Moritz W, Kelm JM, Ullrich ND, Agarkova I, Anson BD, et al. Development and characterization of a scaffold-free 3D spheroid model of induced pluripotent stem cell-derived human cardiomyocytes. Tissue Eng - Part C Methods. 2015;21(8):852-61. https://doi.org/10.1089/ten.tec.2014.0376.

27. Richards DJ, Coyle RC, Tan Y, Jia J, Wong K, Toomer K, et al. Inspiration from heart development: biomimetic development of functional human cardiac organoids. Biomaterials. 2017;142:112-23. https://doi.org/10.1016/j.biomaterials.2017. 07.021 .

28. Nugraha B, Hong X, Mo X, Tan L, Zhang W, Chan PM, et al. Galactosylated cellulosic sponge for multi-well drug safety testing. Biomaterials. 2011;32(29):6982-94. https://doi.org/10. 1016/J.BIOMATERIALS.2011.05.087.

29. Giacomelli E, Meraviglia V, Campostrini G, Cochrane A, Cao $\mathrm{X}$, van Helden RWJ, et al. Human-iPSC-derived cardiac stromal cells enhance maturation in 3D cardiac microtissues and reveal non-cardiomyocyte contributions to heart disease. Cell Stem Cell. 2020;26(6):862-879.e11. https://doi.org/10.1016/j.stem. 2020.05.004

30. Beauchamp P, Jackson CB, Ozhathil LC, Agarkova I, Galindo $\mathrm{CL}$, Sawyer DB, et al. 3D co-culture of hiPSC-derived cardiomyocytes with cardiac fibroblasts improves tissue-like features of cardiac spheroids. Front Mol Biosci. 2020;7:14. https://doi. org/10.3389/fmolb.2020.00014.

31. Lee MO, Jung KB, Jo SJ, Hyun SA, Moon KS, Seo JW, et al. Modelling cardiac fibrosis using three-dimensional cardiac microtissues derived from human embryonic stem cells. J Biol Eng. 2019;13(1):1-17. https://doi.org/10.1186/ s13036-019-0139-6.

32. Polonchuk L, Chabria M, Badi L, Hoflack JC, Figtree G, Davies MJ, et al. Cardiac spheroids as promising in vitro models to study the human heart microenvironment. Sci Rep. 2017;7(1):1-12. https://doi.org/10.1038/s41598-017-06385-8.

33. Archer CR, Sargeant R, Basak J, Pilling J, Barnes JR, Pointon A. Characterization and validation of a human $3 \mathrm{D}$ cardiac microtissue for the assessment of changes in cardiac pathology. Sci Rep. 2018;8(1):1-15. https://doi.org/10.1038/s41598-018-28393-y. This study demonstrated the utility of cardiac spheroid platforms in screening a large panel of drugs for multiple relevant readouts with demonstrable sensitivity and specificity.

34. Kofron CM, Kim TY, Munarin F, Soepriatna AH, Kant RJ, Mende U, et al. A predictive in vitro risk assessment platform for pro-arrhythmic toxicity using human $3 \mathrm{D}$ cardiac microtissues. Sci Rep. 2021;11(1):1-16. https://doi.org/10.1038/ s41598-021-89478-9.

35. Richards DJ, Li Y, Kerr CM, Yao J, Beeson GC, Coyle RC, et al. Human cardiac organoids for the modelling of myocardial infarction and drug cardiotoxicity. Nat Biomed Eng. 2020;4(4):446-62. https://doi.org/10.1038/s41551-020-0539-4.

36. Arai K, Murata D, Takao S, Nakamura A, Itoh M, Kitsuka T, et al. Drug response analysis for scaffold-free cardiac constructs fabricated using bio-3D printer. Sci Rep. 2020;10(1):1-11. https://doi.org/10.1038/s41598-020-65681-y.

37. Thavandiran N, Hale C, Blit P, Sandberg ML, McElvain ME, Gagliardi M, et al. Functional arrays of human pluripotent stem cell-derived cardiac microtissues. Sci Rep. 2020;10(1):1-13. https://doi.org/10.1038/s41598-020-62955-3.
38. Lu HF, Leong MF, Lim TC, Chua YP, Lim JK, Du C, et al. Engineering a functional three-dimensional human cardiac tissue model for drug toxicity screening. Biofabrication. 2017;9(2). https://doi.org/10.1088/1758-5090/aa6c3a.

39. Boudou T, Legant WR, Mu A, Borochin MA, Thavandiran N, Radisic M, et al. A microfabricated platform to measure and manipulate the mechanics of engineered cardiac microtissues. Tissue Eng - Part A. 2012;18(9-10):910-9. https://doi.org/10. 1089/ten.tea.2011.0341.

40. Mills RJ, Titmarsh DM, Koenig X, Parker BL, Ryall JG, QuaifeRyan GA, et al. Functional screening in human cardiac organoids reveals a metabolic mechanism for cardiomyocyte cell cycle arrest. Proc Natl Acad Sci. 2017;114(40):E8372-81. https:// doi.org/10.1073/PNAS.1707316114.

41. Truitt R, Mu A, Corbin EA, Vite A, Brandimarto J, Ky B, et al. Increased afterload augments sunitinib-induced cardiotoxicity in an engineered cardiac microtissue model. JACC Basic to Transl Sci. 2018;3(2):265-76. https://doi.org/10.1016/j.jacbts.2017.12. 007.

42.• Mills RJ, Parker BL, Quaife-Ryan GA, Voges HK, Needham EJ, Bornot A, et al. Drug screening in human PSC-cardiac organoids identifies pro-proliferative compounds acting via the mevalonate pathway. Cell Stem Cell. 2019;24(6):895-907.e6. https://doi.org/ 10.1016/j.stem.2019.03.009. A demonstration of how cardiac microtissues can be used in conjunction with 2D screening platforms to identify $<\mathbf{1 0}$ drug candidates from an initial pool of 5,000.

43. Shum AMY, Che H, Wong AOT, Zhang C, Wu H, Chan CWY, et al. A micropatterned human pluripotent stem cell-based ventricular cardiac anisotropic sheet for visualizing drug-induced arrhythmogenicity. Adv Mater. 2017;29(1):1602448. https://doi. org/10.1002/adma.201602448.

44. Tadano K, Miyagawa S, Takeda M, Tsukamoto Y, Kazusa K, Takamatsu K, et al. Cardiotoxicity assessment using threedimensional vascularized cardiac tissue consisting of fibroblasts and human iPSC-derived cardiomyocytes. Mol Ther - Methods Clin Dev. 2021;22:338. https://doi.org/10.1016/j.omtm.2021.05. 007.

45. Takeda M, Miyagawa S, Ito E, Harada A, Mochizuki-Oda N, Matsusaki M, et al. Development of a drug screening system using three-dimensional cardiac tissues containing multiple cell types. Sci Rep. 2021;11(1):1-12. https://doi.org/10.1038/ s41598-021-85261-y.

46. Eschenhagen T, Fink C, Remmers U, Scholz H, Wattchow J, Weil J, et al. Three-dimensional reconstitution of embryonic cardiomyocytes in a collagen matrix: a new heart muscle model system. FASEB J. 1997;11(8):683-94. https://doi.org/10.1096/ fasebj.11.8.9240969.

47. Hansen A, Eder A, Bönstrup M, Flato M, Mewe M, Schaaf S, et al. Development of a drug screening platform based on engineered heart tissue. Circ Res. 2010;107(1):35-44. https://doi.org/ 10.1161/CIRCRESAHA.109.211458.

48. Nunes SS, Miklas JW, Liu J, Aschar-Sobbi R, Xiao Y, Zhang B, et al. Biowire: A platform for maturation of human pluripotent stem cell-derived cardiomyocytes. Nat Methods. 2013;10(8):7817. https://doi.org/10.1038/nmeth.2524.

49. Feric NT, Pallotta I, Singh R, Bogdanowicz DR, Gustilo MM, Chaudhary KW, et al. Engineered cardiac tissues generated in the Biowire II: a platform for human-based drug discovery. Toxicol Sci. 2019;172(1):89-97. https://doi.org/10.1093/toxsci/ kfz168.

50. Mannhardt I, Eder A, Dumotier B, Prondzynski M, Kr-amer E, Traebert M, et al. Blinded contractility analysis in hipsccardiomyocytes in engineered heart tissue format: comparison 
with human atrial trabeculae. Toxicol Sci. 2017;158(1):164-75. https://doi.org/10.1093/toxsci/kfx081.

51. Saleem U, Meer BJV, Katili PA, Yusof NANM, Mannhardt I, Garcia AK, et al. Blinded, multicenter evaluation of drug-induced changes in contractility using human-induced pluripotent stem cell-derived cardiomyocytes. Toxicol Sci. 2020;176(1):103-23. https://doi.org/10.1093/toxsci/kfaa058.

52. Ronaldson-Bouchard K, Ma SP, Yeager K, Chen T, Song LJ, Sirabella D, et al. Advanced maturation of human cardiac tissue grown from pluripotent stem cells. Nature. 2018;556(7700):239-43. https://doi.org/10.1038/s41586-018-0016-3. An electrical stimulation protocol produced among the most structurally and electrophysiologically mature engineered cardiac tissues to date.

53. Zhao Y, Rafatian N, Feric NT, Cox BJ, Aschar-Sobbi R, Wang $\mathrm{EY}$, et al. A platform for generation of chamber-specific cardiac tissues and disease modeling. Cell. 2019;176(4):913-927.e18. https://doi.org/10.1016/j.cell.2018.11.042.

54. Lemme M, Ulmer BM, Lemoine MD, Zech ATL, Flenner F, Ravens U, et al. Atrial-like engineered heart tissue: an in vitro model of the human rtrium. Stem Cell Reports. 2018;11(6):137890. https://doi.org/10.1016/j.stemcr.2018.10.008.

55. Wang EY, Kuzmanov U, Smith JB, Dou W, Rafatian N, Lai BFL, et al. An organ-on-a-chip model for pre-clinical drug evaluation in progressive non-genetic cardiomyopathy. J Mol Cell Cardiol. 2021;160:97-110. https://doi.org/10.1016/j.yjmcc.2021.06.012.

56. Stoehr A, Neuber C, Baldauf C, Vollert I, Friedrich FW, Flenner F, et al. Automated analysis of contractile force and $\mathrm{Ca} 2+$ transients in engineered heart tissue. AJP Hear Circ Physiol. 2014;306(9):H135363. https://doi.org/10.1152/ajpheart.00705.2013.

57. Mannhardt I, Saleem U, Benzin A, Schulze T, Klampe B, Eschenhagen T, et al. Automated contraction analysis of human engineered heart tissue for cardiac drug safety screening. J Vis Exp. 2017;2017(122):55461. https://doi.org/10.3791/55461.

58. Bielawski KS, Leonard A, Bhandari S, Murry CE, Sniadecki NJ. Real-rime force and frequency analysis of engineered human heart tissue derived from induced pluripotent stem cells using magnetic sensing. Tissue Eng Part C. 2016;22(10):932-40. https://doi.org/10.1089/ten.tec.2016.0257.

59. Tiburcy M, Meyer T, Soong PL, Zimmermann WH. Collagen-based engineered heart muscle. Methods Mol Biol. 2014;1181:167-76. https://doi.org/10.1007/978-1-4939-1047-2_15.

60. Goldfracht I, Efraim Y, Shinnawi R, Kovalev E, Huber I, Gepstein A, et al. Engineered heart tissue models from hiPSC-derived cardiomyocytes and cardiac ECM for disease modeling and drug testing applications. Acta Biomater. 2019;92:145-59.

61. Goldfracht I, Protze S, Shiti A, Setter N, Gruber A, Shaheen $\mathrm{N}$, et al. Generating ring-shaped engineered heart tissues from ventricular and atrial human pluripotent stem cell-derived cardiomyocytes. Nat Commun. 2020;11(1):1-15. https://doi.org/ 10.1038/s41467-019-13868-X.

62. MacQueen LA, Sheehy SP, Chantre CO, Zimmerman JF, Pasqualini FS, Liu X, et al. A tissue-engineered scale model of the heart ventricle. Nat Biomed Eng. 2018;2(December):930-41. https://doi.org/10. 1038/s41551-018-0271-5.

63. Li RA, Keung W, Cashman TJ, Backeris PC, Johnson BV, Bardot $\mathrm{ES}$, et al. Bioengineering an electro-mechanically functional miniature ventricular heart chamber from human pluripotent stem cells. Biomaterials. 2018;163:116-27. https://doi.org/10.1016/j.biomaterials.2018. 02.024 .

64.• Keung W, Chan PKW, Backeris PC, Lee EK, Wong N, Wong AOT, et al. Human cardiac ventricular-like organoid chambers and tissue strips from pluripotent stem cells as a two-tiered assay for inotropic responses. Clin Pharmacol Ther. 2019;106(2):40214. https://doi.org/10.1002/cpt.1385. A demonstration of the improved sensitivity and positive inotropy of higher-order cardiac chambers as compared to cardiac strips.

65. Williams NP, Rhodehamel M, Yan C, Smith AST, Jiao A, Murry $\mathrm{CE}$, et al. Engineering anisotropic 3D tubular tissues with flexible thermoresponsive nanofabricated substrates. Biomaterials. 2020;240: 119856. https://doi.org/10.1016/J.BIOMATERIALS. 2020.119856.

66. Van Den Berg CW, Okawa S, Chuva De Sousa Lopes SM, Van Iperen L, Passier R, Braam SR, et al. Transcriptome of human foetal heart compared with cardiomyocytes from pluripotent stem cells. Dev. 2015;142(18):3231-8. https://doi.org/10.1242/ dev. 123810

67. Davis RP, van den Berg CW, Casini S, Braam SR, Mummery CL. Pluripotent stem cell models of cardiac disease and their implication for drug discovery and development. Trends in Molecular Medicine. Elsevier Current Trends; 2011;17:47584. https://doi.org/10.1016/j.molmed.2011.05.001.

68. Ulmer BM, Eschenhagen T. Human pluripotent stem cell-derived cardiomyocytes for studying energy metabolism. Biochimica et Biophysica Acta - Mol Cell Res. Elsevier B.V. 2020;1867:118471. https://doi. org/10.1016/j.bbamcr.2019.04.001.

69. Pioner JM, Racca AW, Klaiman JM, Yang K-C, Guan X, Pabon $\mathrm{L}$, et al. Isolation and mechanical measurements of myofibrils from human induced pluripotent stem cell-derived cardiomyocytes. Stem Cell Reports. 2016;6(6):885-96. https://doi.org/10. 1016/j.stemcr.2016.04.006.

70. Yang X, Rodriguez ML, Leonard A, Sun L, Fischer KA, Wang $\mathrm{Y}$, et al. Fatty acids enhance the maturation of cardiomyocytes derived from human pluripotent stem cells. Stem Cell Reports. 2019;13(4):657-68. https://doi.org/10.1016/j.stemcr.2019.08.013.

71. Yang X, Rodriguez M, Pabon L, Fischer KA, Reinecke H, Regnier $\mathrm{M}$, et al. Tri-iodo-1-thyronine promotes the maturation of human cardiomyocytes-derived from induced pluripotent stem cells. J Mol Cell Cardiol. 2014;72:296-304. https://doi.org/10.1016/j. yjmcc.2014.04.005.

72. Kuppusamy KT, Jones DC, Sperber H, Madan A, Fischer KA, Rodriguez ML, et al. Let-7 family of microRNA is required for maturation and adult-like metabolism in stem cell-derived cardiomyocytes. Proc Natl Acad Sci. 2015;112(21):E2785-94. https:// doi.org/10.1073/pnas.1424042112.

73. Miklas JW, Clark E, Levy S, Detraux D, Leonard A, Beussman K, et al. TFPa/HADHA is required for fatty acid beta-oxidation and cardiolipin re-modeling in human cardiomyocytes. Nat Commun. 2019;10(1):1-21. https://doi.org/10.1038/s41467-019-12482-1.

74. Leonard A, Bertero A, Powers JD, Beussman KM, Bhandari $\mathrm{S}$, Regnier M, et al. Afterload promotes maturation of human induced pluripotent stem cell derived cardiomyocytes in engineered heart tissues. J Mol Cell Cardiol. 2018;118:147-58. https://doi.org/10.1016/j.yjmcc.2018.03.016.

75. Bliley JM, Vermeer MCSC, Duffy RM, Batalov I, Kramer D, Tashman JW, et al. Dynamic loading of human engineered heart tissue enhances contractile function and drives desmosome-linked disease phenotype. bioRxiv. 2020. https://doi.org/10.1101/2020.05. 25.111690 .

76. Lu K, Seidel T, Cao-Ehlker X, Dorn T, Nazeer Batcha AM, Schneider $\mathrm{CM}$, et al. Progressive stretch enhances growth and maturation of 3D stem-cell-derived myocardium. 2021;11(13):13. https://doi.org/10. 7150/thno.54999.

77. Stoehr A, Neuber C, Baldauf C, Vollert I, Friedrich FW, Flenner $\mathrm{F}$, et al. Automated analysis of contractile force and $\mathrm{Ca} 2+$ transients in engineered heart tissue. Am J Physiol Circ Physiol. 2014; 306(9):H1353-63. https://doi.org/10.1152/ajpheart.00705.2013.

78. Mannhardt I, Saleem U, Mosqueira D, Loos MF, Ulmer BM, Lemoine MD, et al. Comparison of 10 control hPSC lines for 
drug screening in an engineered heart tissue format. Stem Cell Reports. 2020;15(4):983-98. https://doi.org/10.1016/j.stemcr. 2020.09.002.

79. Hoes MF, Bomer N, van der Meer P. Concise review: The current state of human in vitro cardiac disease modeling: a focus on gene editing and tissue engineering. Stem Cells Transl Med. 2019;8(1):66-74. https://doi.org/10.1002/sctm.18-0052.

80. Lock R, Asafen H Al, Fleischer S, Tamargo M, Zhao Y, Radisic $\mathrm{M}$, et al. A framework for developing sex-specific engineered heart models. Nat Rev Mater. 2021;1. https://doi.org/10.1038/ S41578-021-00381-1.

81. Williams MAC, Mair DB, Lee W, Lee E, Kim DH. Engineering three-dimensional vascularized cardiac tissues. Tissue Eng Part B Rev. 2021. https://doi.org/10.1089/ten.teb.2020.0343.

82. Osaki T, Sivathanu V, Kamm RD. Vascularized microfluidic organ-chips for drug screening, disease models and tissue engineering. Curr Opin Biotechnol. 2018;52:116-23. https://doi.org/ 10.1016/J.COPBIO.2018.03.011.

83. Lai BFL, Huyer LD, Lu RXZ, Drecun S, Radisic M, Zhang B. InVADE: Integrated vasculature for assessing dynamic events. Adv Funct Mater. 2017;27(46):1703524. https://doi.org/10.1002/ adfm. 201703524.
84. Picollet-D'hahan N, Zuchowska A, Lemeunier I, Le Gac S. Multiorgan-on-a-chip: A systemic approach to model and decipher inter-organ communication. Trends in Biotechnol. Elsevier Curr Trends. 2021;39:788-810. https://doi.org/10.1016/j.tibtech. 2020.11.014.

85. Shen S, Sewanan LR, Jacoby DL, Campbell SG. Danicamtiv enhances systolic function and frank-starling behavior at minimal diastolic cost in engineered human myocardium. J Am Heart Assoc. 2021;10(12):20860. https://doi.org/10.1161/JAHA.121.020860.

86. Wong AOT, Gurung B, Wong WS, Mak SY, Tse WW, Li CM, et al. Adverse effects of hydroxychloroquine and azithromycin on contractility and arrhythmogenicity revealed by human engineered cardiac tissues. J Mol Cell Cardiol. 2021;153:106-10. https://doi.org/10.1016/j.yjmcc.2020.12.014.

Publisher's Note Springer Nature remains neutral with regard to jurisdictional claims in published maps and institutional affiliations. 